\title{
Synthesis of multi-substituted furan rings: the role of silicon
}

\author{
Brian A. Keay
}

Department of Chemistry, University of Calgary, Calgary, Alberta, Canada, T2N 1N4. E-mail: keay@ucalgary.ca

\section{Received 3rd December 1998}

Silanes have proven extremely useful for the synthesis of multi-substituted furan rings. Their judicious placement on a furan ring can be used to control either the placement of new groups around the furan ring or the silane can be directly replaced via an ipso-substitution. This paper will briefly review the role silicon has played in the preparation of furan rings having a variety of substituent patterns.

\section{Introduction}

Compounds that have a multi-substituted furan ring as their main component or have a furan ring fused with other rings have exhibited a vast array of activity.' Thus, the design of synthetic routes towards furan rings having specific substitution patterns has interested chemists for decades. ${ }^{2}$ Of the many strategies developed for the preparation of a multi-substituted furan ring, one of the simplest involves starting with either furan itself or a mono-substituted furan followed by the introduction of functional groups at various positions around the furan ring. This approach, however, has some drawbacks. First, the introduction of functional groups into the 3- and 4-positions is difficult as furan rings lithiate and add electrophiles preferentially at the 2 and 5 positions. Second, placement of a group at C-3 results in the 2 and 5 positions becoming regioisomeric so that either regioselective methods or blocking groups must be used to introduce new moieties. ${ }^{2}$ The use of an organosilyl group as a blocking group has been the most useful in furan chemistry. Not only are they easy to introduce and remove, but they can be

Brian A. Keay was born in 1955 and received his $P h D$ degree in 1983 from the University of Waterloo (Canada) working with Professor R. Rodrigo. After an NSERC postdoctoral fellowship with Professor E. Piers (University of British Columbia Canada), he joined the faculty at the University of Windsor (Canada) as an assistant Professor. In 1989, he moved to the University of Calgary where he

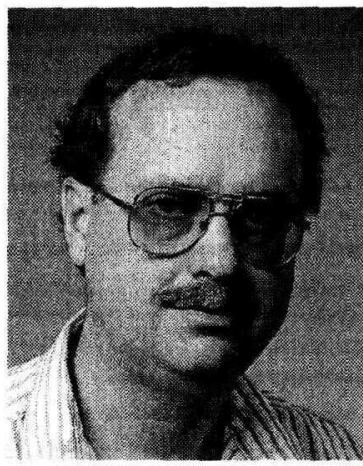
is now a Professor. In 1996, he was awarded the Merck Frosst Centre for Therapeutic Research Lecture Award by the Canadian Society for Chemistry. His research interests in clude the design and synthesis of asymmetric ligands for use with Lewis acids and transition metals, palladium-catalyzed polyene cyclizations, intramolecular Diels-Alder reactions of furans and the synthesis of natural products. replaced by electrophiles via an ipso-substitution. In addition, their size has had a pronounced influence on the regioselectivity for introduction of other groups on the furan ring and on some rearrangements. This paper will briefly review the role organosilyl groups have played in the synthesis of multisubstituted furan rings. This will not be an exhaustive review, but will concentrate on how silyl groups have been used to prepare specific substitution patterns with furan rings.

\section{The role of silyl groups on furan rings}

In 1948 Benkeser and Currie reported ${ }^{3}$ not only the introduction of a trimethylsilyl group at the $\mathrm{C}-2$ position of furan, but that the silyl group could later be replaced by a bromine atom via an ipso-substitution (Scheme 1). Treatment of furan (1) with BuLi

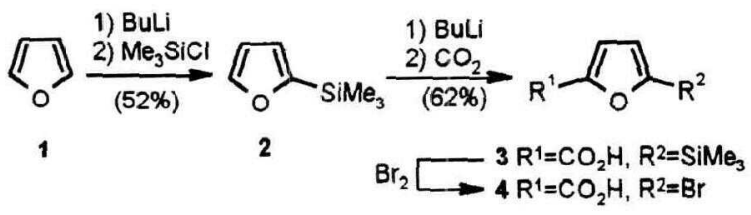

Scheme 1

followed by the addition of trimethylsilyl chloride provided 2-(trimethylsilyl)furan (2), which when treated again with BuLi followed by the addition of $\mathrm{CO}_{2}$ gave 5-(trimethylsilyl)2-furoic acid (3). Subsequent treatment of 3 with $\mathrm{Br}_{2}$ in $\mathrm{CCl}_{4}$ gave 5-bromo-2-furoic acid (4, no yield given). This work also confirmed that furan (1) lithiated at the C-2 position when treated with $\mathrm{BuLi}$. To my knowledge this was the first report of the introduction of an organosilyl group onto a furan ring and the first example of an ipso-substitution on a furan ring.

In order to introduce functional groups regioselectively around the furan ring, it is sometimes prudent to use a group to block a particular position. Organosilyl moieties have been used as blocking groups as they are easily introduced and removed. Carpenter and Chadwick ${ }^{4}$ required the synthesis of 2,3-disubstituted furan rings, but as mentioned above, the introduction of groups into the 3 position of a furan ring is difficult. They achieved the introduction of a group at the C-3 position of 2 -furoic acid (5) by first blocking the C-5 position with a trimethylsilyl group, which afforded acid 3, according to the protocol developed by Knight ${ }^{5}$ (Scheme 2). Treatment of 3 with $\mathrm{BuLi}\left(\mathrm{THF},-78^{\circ} \mathrm{C}, 0.5 \mathrm{~h}\right.$ ) resulted in a regiospecific lithiation at $\mathrm{C}-3$. Quenching the anion with $\mathrm{CO}_{2}$ followed by an acidic workup gave 6. The trimethylsilyl group was then removed to give furan-2,3-dicarboxylic acid (7). The yield for the overall sequence was $78 \%$. A similar sequence (but using different reagents) was also reported by Chadwick ${ }^{6}$ using 2-(4,4-dimethyl-4,5-dihydro-1,3-oxazol-2-yl)furan 8 instead of 2 -furoic 


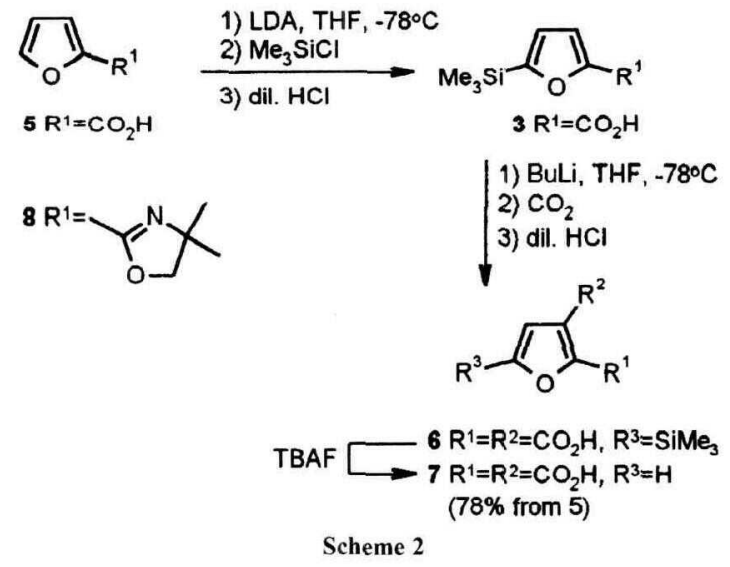

acid (5) and has been expanded upon by Gilchrist ${ }^{7}$ and Queguiner. ${ }^{8}$

Keay and co-workers ${ }^{9,10}$ have developed a strategy for the synthesis of a variety of polysubstituted furan rings by taking advantage of the silyl groups: 1) ability to be a blocking group; 2) tendency to undergo silyl migrations; and 3) size. In this manner they have been able to develop routes towards the synthesis of 2,3-, 2,3,4-, 2,4-, and the more difficult 3,4-substituted furan systems starting from a common 3 -substituted furan ring.

First it was shown that a variety of silyl protected 3-hydroxymethylfurans 9 undergo an intramolecular $[1,4] \mathrm{O} \rightarrow \mathrm{C}$ silyl migration to produce 2,3-disubstituted furans $\mathbf{1 0}$ in good to excellent yield by treating 9 with a mixture of $\mathrm{BuLi}$ and HMPA in THF (Scheme 3). ${ }^{9}$ Treatment of $\mathbf{1 0}$ with 2.2 equiv. of BuLi in

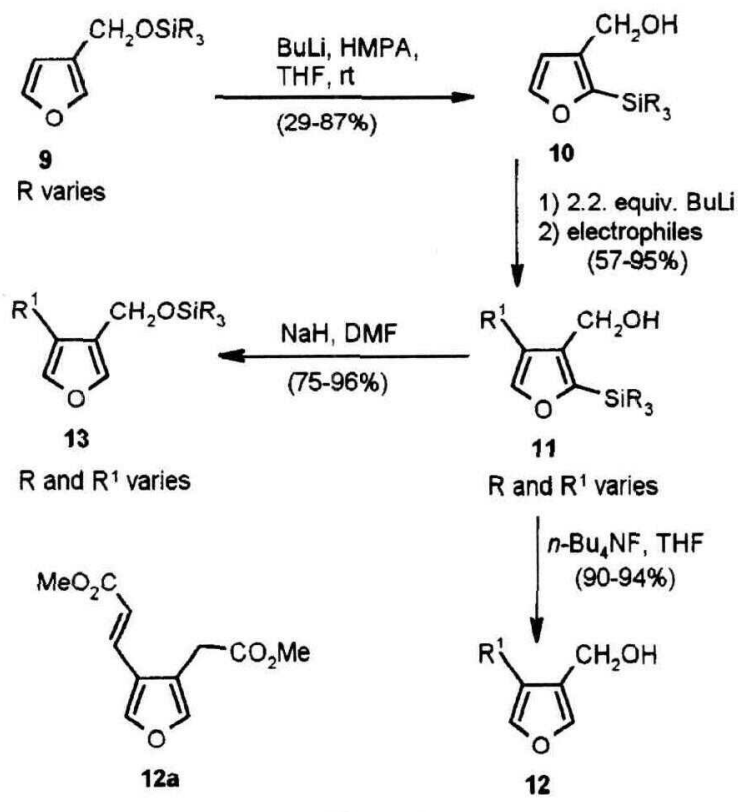

Scheme 3

DME resulted in a regiospecific C-4 lithiation. Quenching the anion with a variety of electrophiles provided 2,3,4-trisubstituted furans 11 in good to excellent yields. ${ }^{10}$ To our knowledge, this was the first example of a direct $\mathrm{C}-4$ lithiation in the presence of an unprotected C-5 position on a furan ring. This regiospecific lithiation was a result of the presence of the silyl blocking group at $\mathrm{C}-2$ and also was a result of the size of the silyl group forcing the hydroxymethyl group to reside very close to the $\mathrm{C}-4$ hydrogen atom so that when BuLi was added, the $\mathrm{C}-4$ hydrogen atom was preferentially abstracted instead of the $\mathrm{C}-5$ hydrogen atom. This chemistry also provided a route to directly access 3,4-disubstituted furan rings, which of all the possible furan patterns, are the most difficult to prepare. ${ }^{2}$ Treatment of $\mathbf{1 1}$ with TBAF in THF resulted in the direct removal of the C-2 silyl group to provide 3,4-substituted furans 12. Interestingly, the silyl group could be reused to protect the hydroxy group at $\mathrm{C}-3$ by treating $\mathbf{1 1}$ with $\mathrm{NaH}$ in DMF. This resulted in an intramolecular $[1,4] \mathrm{C} \rightarrow \mathrm{O}$ silyl migration to form 13. ${ }^{9}$ The complete sequence in Scheme 3 illustrates the importance and usefulness of a silyl group. This approach was used recently by Scott and co-workers to prepare furan 12a. ${ }^{\prime \prime}$

It is interesting to note that if the hydroxymethyl group of $\mathbf{1 0}$ is protected as a triethylsilyl12 or tert-butyldimethylsilyl group, ${ }^{13}$ to give $\mathbf{1 4}$, and $\mathbf{1 4}$ is then treated with 1.3 equiv. of BuLi, lithiation occurs exclusively at C-5 (Scheme 4). Trapping

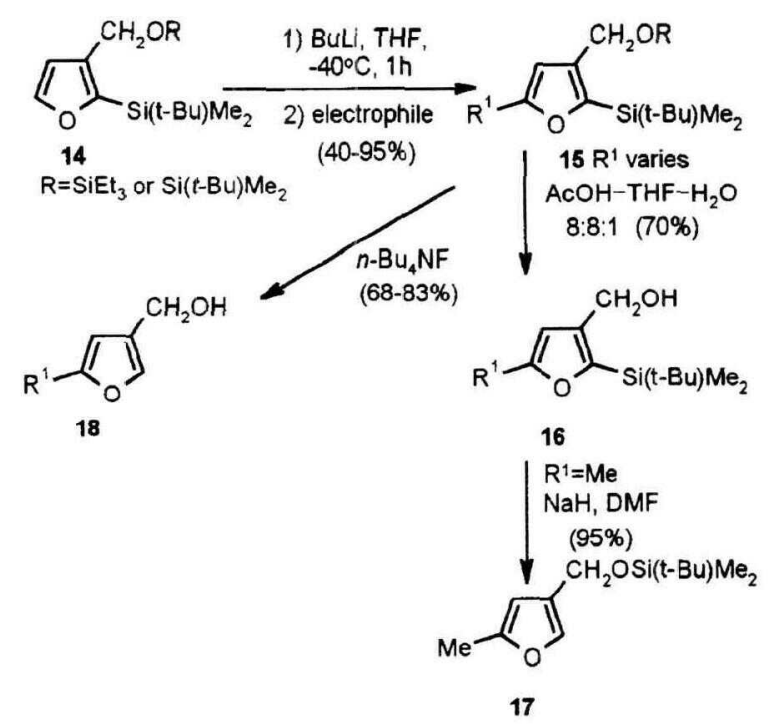

Scheme 4

the C-5 anion with electrophiles provided 2,3,5-trisubstituted furans 15. When the triethylsilyl group was used, it could be selectively removed $\left(\mathrm{AcOH}-\mathrm{THF}-\mathrm{H}_{2} \mathrm{O} ; 8: 8: 1\right)$ to provide alcohol 16. The hydroxy group in $\mathbf{1 6}$ could be re-protected by the silyl group at $\mathrm{C}-2$ via a $[1,4] \mathrm{C} \rightarrow \mathrm{O}$ silyl migration thereby providing access to 2,4-disubstituted furan rings 17 . In addition, both silyl groups could be removed by treatment with TBAF in THF to provide furans like 18. Recently Katsumura used this chemistry to prepare tetrasubstituted furan rings as precursors towards butenolides. ${ }^{14}$

One of the more difficult transformations with 2- or 3 -furaldehyde is their direct oxidation to 2-or 3-furoic acid, since the furan ring is very sensitive to acidic reagents and normally most oxidations of aldehydes to acids involve using acidic reagents. Thus attempts to oxidize $\mathbf{1 2}$ to the corresponding furoic acid failed. To overcome this difficulty, Keay and coworkers showed that silyl esters of 3-furoic acid 19 also undergo a $[1,4] \mathrm{O} \rightarrow \mathrm{C}$ silyl migration to provide 2 -silylated-3-furoic acids $\mathbf{2 0}$ in moderate yield (Scheme 5). ${ }^{9}$ Acids $\mathbf{2 0}$ when treated with $\mathrm{BuLi}$ resulted in a regiospecific $\mathrm{C}-4$ lithiation. Trapping the anion with a variety of electrophiles provided 2,4-disubstituted-3-furoic acids 21 in good to excellent yield. ${ }^{10}$ The silyl group was easily removed with TBAF and the acid converted to the methyl ester (for ease of workup) by treatment with diazomethane to provide 4-substituted-3-furoic esters 22 . Quayle and co-workers have used the silyl migration of silylesters 19 to prepare 2-silylated tetrahydrofurans $\mathbf{2 3} .{ }^{15}$ 


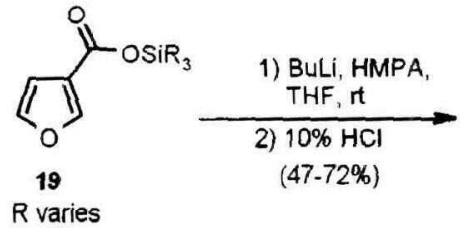

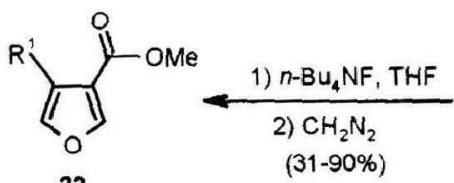<smiles>[B]C1OCC[C@@H]1C(=O)OC</smiles>

Scheme 5

In order to illustrate the usefulness of our silyl strategy, we investigated the introduction of other groups into the C-4 position of furan 10 (Scheme 6). ${ }^{16}$ The C-4 anion of furan 10

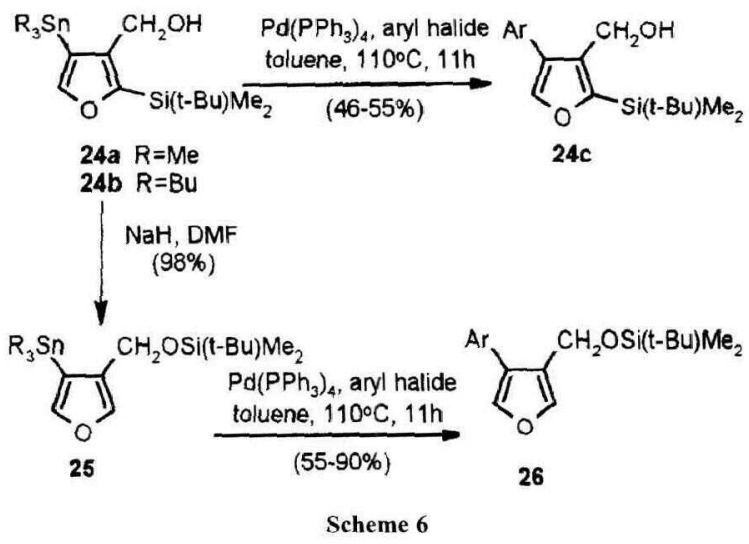

could be trapped with tributyl- or trimethylstannyl chloride providing furans $\mathbf{2 4 a}$ and $\mathbf{2 4 b}$. The direct Stille coupling with $\mathbf{2 4 a}$ or $\mathbf{b}$ provided disappointing yields of furans $\mathbf{2 4 c}$; however, when the silyl group at C-2 was migrated to the hydroxy group at C-3 (to give 25), the Stille coupling proceeded in yields ranging from $55-90 \%$ to provide 26 . This gave access to a variety of 3,4-disubstituted furans in which one of the substituents was an aromatic ring.

Since the Stille coupling with $\mathbf{2 4 a}$ and $\mathbf{2 4 b}$ was sluggish, we investigated trying a Suzuki coupling. This required the preparation of boronic acid 27 (Scheme 7). Unfortunately, the isolation of boronic acid $\mathbf{2 7}$ after quenching the C-4 anion of $\mathbf{1 0}$ with trimethylborate was extremely difficult. Thus, we developed an in situ variant of the Suzuki reaction in which the boronic acid component does not have to be isolated. ${ }^{17,18}$ The reaction is easy to perform. Trimethylborate is added to the anion and the reaction is stirred for $\mathrm{I} h$. Then instead of working up the mixture, an aryl or vinyl halide or triflate is added, along with $\mathrm{Pd}\left(\mathrm{PPh}_{3}\right)_{4}$, water, and $\mathrm{Na}_{2} \mathrm{CO}_{3}$ and the mixture is refluxed for 1-20 h. A variety of products $\mathbf{2 8}$ were isolated in yields ranging from $20-93 \%$. The in situ variant of the Suzuki reaction is a general reaction and is not limited to just furan systems; a variety of vinyl and aryl carbanions can be treated in a similar manner. ${ }^{18}$

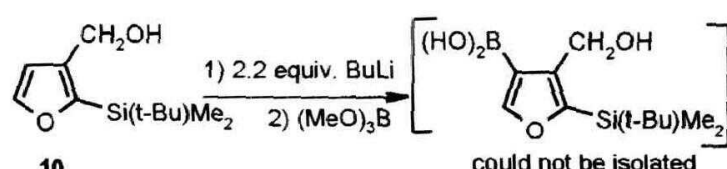

10

could not be isolated

27

$\mathrm{Pd}\left(\mathrm{PPh}_{3}\right)_{4}$, aryl or

vinyl halide or triflate

DME, $\mathrm{H}_{2} \mathrm{O}, 80^{\circ} \mathrm{C}$

(20-93\% from 17)<smiles>[R]c1coc([SiH2]C(C)(C)C)c1CO</smiles>

28

$\mathrm{R}=$ aryl or vinyl

Scheme 7

Finally, a tetrasubstituted furan ring can be generated very easily by the metalation of furan $\mathbf{2 9}$ (Scheme 8), which was prepared using the strategy illustrated in Scheme 7. For

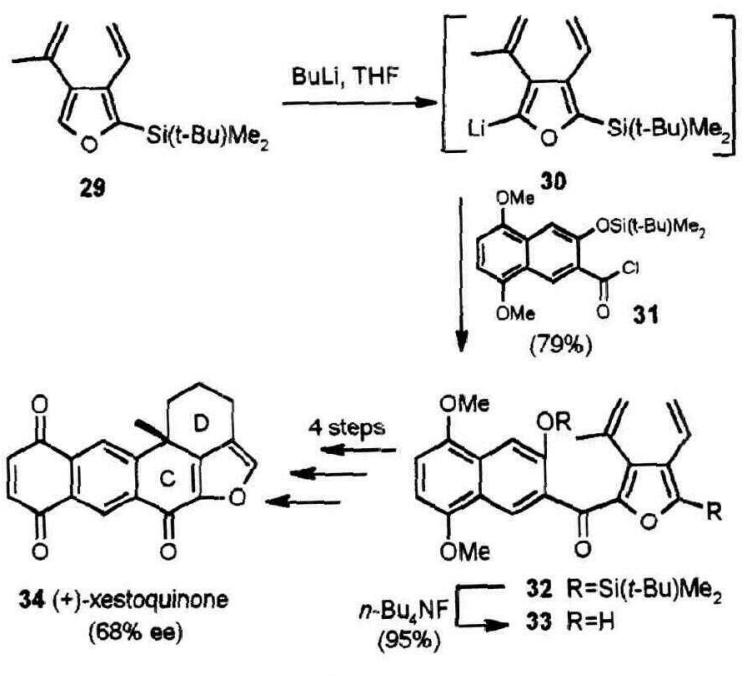

Scheme 8

example, furan 29, when treated with $\mathrm{BuLi}$, provided the $\mathrm{C}-5$ anion 30, which when quenched with acid chloride $\mathbf{3 1}$ provided tetrasubstituted furan $\mathbf{3 2}$. Both silyl groups in $\mathbf{3 2}$ were easily removed to provide a 2,3,4-tribsubstituted furan 33, which is structurally different than the 2,3,4-trisubstituted furan that can be prepared via Schemes 3, 6 and 7 .

Furan 33 was required as an intermediate towards the synthesis of $(+)$-xestoquinone $(\mathbf{3 4}$, Scheme 8$) .{ }^{19}$ Conversion of the hydroxy group in $\mathbf{3 3}$ into a triflate (quantitative) followed by an asymmetric palladium-catalyzed polyene cyclization formed rings $\mathrm{C}$ and $\mathrm{D}(82 \%$ yield, $68 \%$ ee). Two additional steps afforded $(+)-34$.

Tanaka and co-workers ${ }^{20}$ have been investigating how silylated furan rings behave towards conditions that favor ipsosubstitution. Treatment of 35a or 35b (prepared via Diels-Alder chemistry), with sulfuryl chloride, bromine or iodine monochloride afforded $36 \mathrm{a}-\mathrm{e}$ in yields ranging from $64-76 \%$ (Scheme 9). In related work, they also reported an interesting $[1,2] \mathrm{C} \rightarrow \mathrm{C}$ sily] migration. ${ }^{21}$ When furan 37 was treated with sulfuryl chloride in acetonitrile, the expected ipso-substituted product 38 was obtained. ${ }^{22}$ However, when the solvent was changed to $\mathrm{CH}_{2} \mathrm{Cl}_{2}$ a $24: 76$ mixture of $\mathbf{3 8 : 3 9}$ was obtained in which the major compound 39 had the silyl group at C-4 and a 


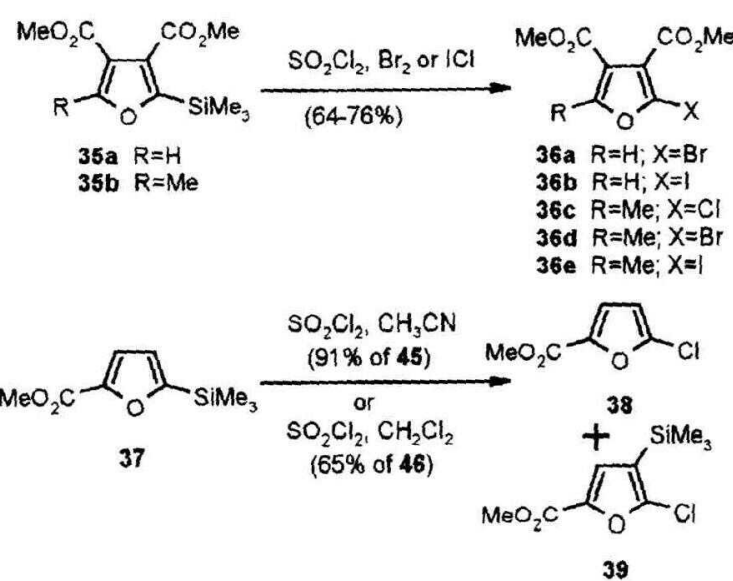

Scheme 9

chlorine atom at C-5. This is the first example of a $[1,2] \mathrm{C} \rightarrow \mathrm{C}$ silyl migration with a furan ring.

Wong and co-workers have reported some very interesting furan chemistry starting from 3,4-bis(trimethylsilyl)furan (42, Scheme 10). A Diels-Alder reaction between oxazole $\mathbf{4 0}$ and<smiles>CC#C[C+]c1cocn1</smiles>

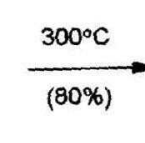

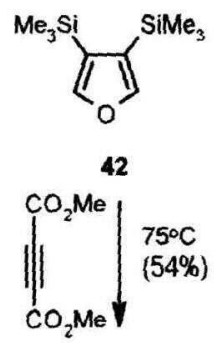<smiles>CC(=O)c1cocc1C(C)=O</smiles>

43

Scheme 10

bis(trimethylsilyl)acetylene (41) followed by a retro-DielsAlder reaction of the initial adduct liberates benzenenitrile and furan 42.23 From this relatively simple furan, they have designed routes to 3,4-,2,4-, and 2,3,5-substituted furan rings. For example, heating $\mathbf{4 2}$ with acetylenic dienophiles, like dimethyl acetylenedicarboxylate, provided 3,4-disubstituted furans like $\mathbf{4 3}$ ( $54 \%$ yield)

Wong has extended this chemistry by developing a route to the preparation of 3,4-disubstituted furans starting with $\mathbf{4 2}$ by performing two successive sequences. ${ }^{24,25}$ The first reaction of each sequence was an ipso-substitution with one equivalent of $\mathrm{BCl}_{3}$ to provide boroxine 44 in $98 \%$ yield (Scheme 11). The second reaction in the sequence was a Suzuki coupling between $\mathbf{4 4}$ and a variety of aryl and heteroaryl halides to provide $\mathbf{4 5}$. Repeating this sequence on $\mathbf{4 5}$ provided 3,4-disubstituted furans 46 in which two aryl or heteroaryl groups are present (they could be the same group or could be different).

A year later, Wong ${ }^{26}$ expanded upon this sequence by showing that when $\mathbf{4 4}$ or $\mathbf{4 7}$ was treated with o-bis(bromomethyl)arenes in the presence of a palladium catalyst, 48 and/or 49 were formed in various ratios depending on the arene that was used. For example, when the reaction between 44 and 2,3-bis(bromomethyl)quinoxaline (50) was performed, only 49<smiles>[R]c1cocc1[R]</smiles><smiles></smiles>
$(98 \%)$<smiles>COc1cocc1B1OB(c2cocc2S(C)(=O)=O)OB(c2cocc2S(C)(=O)=O)O1</smiles>

44

$\mathrm{R}^{1} \mathrm{X}, \mathrm{Pd}\left(\mathrm{PPh}_{3}\right)_{4}$, PhMe-MeOH $120^{\circ} \mathrm{C}, 14-18 \mathrm{~h}$

1) $\mathrm{BCl}_{3,}-78^{\circ} \mathrm{C}$ $(45-97 \%)$

2) $5 \% \mathrm{HCl}(41-61 \%) \mathrm{Me}_{3} \mathrm{~S}$ 3) $\mathrm{R}^{2} \mathrm{X}, \mathrm{Pd}\left(\mathrm{PPh}_{3}\right)_{4}$. $\mathrm{PhMe}-\mathrm{MeOH}$ $120^{\circ} \mathrm{C}, 2.5-5.5 \mathrm{~h}$ 46 $(61-92 \%)$

Scheme 11

was obtained in $90 \%$ yield. He used this unique result with quinoxaline $\mathbf{5 0}$ to his advantage by homo-coupling a variety of boroxines $47\left(\mathrm{R}=\mathrm{CH}_{2} \mathrm{C}_{6} \mathrm{H}_{4}-p-\mathrm{CO}_{2} \mathrm{Me}, \mathrm{n}-\mathrm{Bu}, \mathrm{C}_{6} \mathrm{H}_{4}-\mathrm{O}-\mathrm{Me}\right.$, $\mathrm{C}_{6} \mathrm{H}_{4}-p-\mathrm{Me}$, and $\mathrm{C}_{6} \mathrm{H}_{5}$ ) in yields ranging from $52-80 \%$. One interesting application of the chemistry shown in Scheme 12

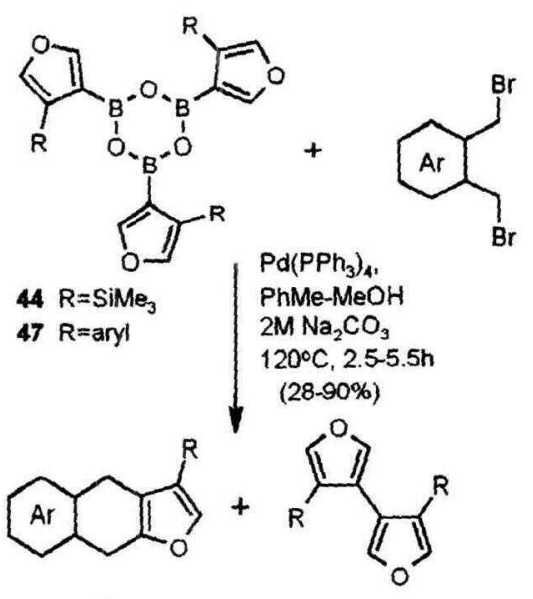<smiles>BrCc1nc2ccccc2nc1CBr</smiles>

Scheme 12

was to take compound $49\left(\mathrm{R}=\mathrm{SiMe}_{3}\right)$ and treat it with one equivalent of $\mathrm{BCl}_{3}$ to form the boroxine $\mathbf{5 1}$ (Scheme 13). When $\mathbf{5 1}$ was homo-coupled in the presence of $\mathbf{5 0}$ and a palladium catalyst, quaterfuran 52 was formed. A repeat of the sequence provided octifuran $\mathbf{5 3}$

In addition to the above interesting chemistry, Wong and coworkers ${ }^{27}$ have reported a mono-ipso-iodination of $\mathbf{4 2}$ and its use in the preparation of 3,4-disubsituted furan rings (Scheme 14). Treatment of $\mathbf{4 2}$ with iodine in the presence of silver trifluoroacetate provided furan $\mathbf{5 4}$ in $80 \%$ yield. With $\mathbf{5 4}$ in hand, the authors performed Heck, Stille and Suzuki reactions that provided a large number of 3-substituted-4-(trimethylsilyl)furans 55. The second trimethylsilyl group was then replaced using Wong's boroxine protocol (see Scheme 11) to 

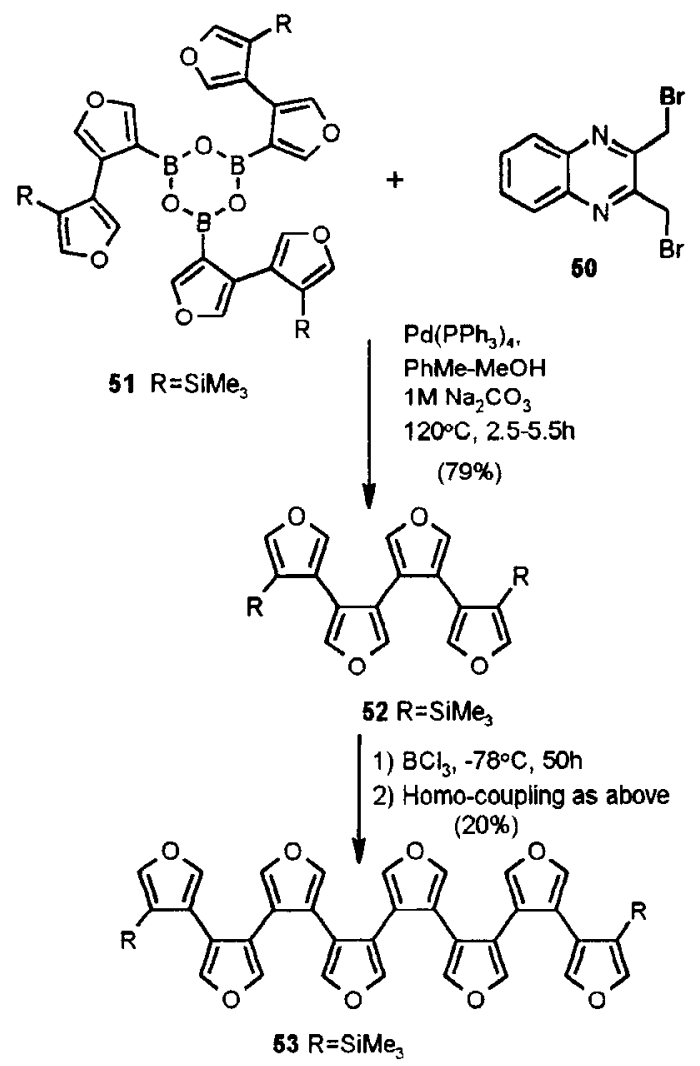

Scheme 13

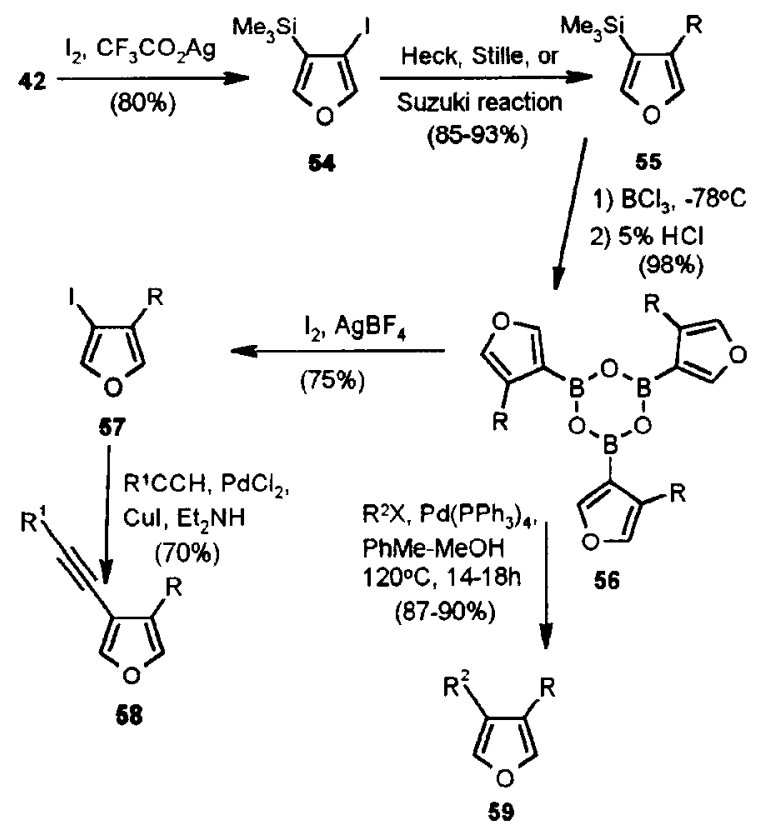

Scheme 14

give 56. Furan 56 could be treated with $\mathrm{I}_{2}$ in the presence of $\mathrm{AgBF}_{4}$ to provide iodide 57, which was used to prepare acetylenic furans like 58. Or, 56 could be used directly in a Suzuki coupling to give 3,4-disubstituted furans 59. The monoreplacement of the trimethylsilyl groups in $\mathbf{4 2}$ clearly expands the scope of the use of furan $\mathbf{4 2}$ in synthesis.
Most recently, Wong and colleagues ${ }^{28}$ have reported new strategies towards the synthesis of 2,3-, 2,4-, 2,3,4-, and $2,3,5$-substituted furan rings. The approach towards the preparation of 2,3-disubstituted furan rings was developed by observing that when furan 42 is heated in a sealed tube at $160^{\circ} \mathrm{C}$ in the presence of trifluoroacetic anhydride containing a catalytic amount of trifluoroacetic acid, one of the silyl groups undergoes a $[1,2] \mathrm{C} \rightarrow \mathrm{C}$ silyl migration to provide 2,4-bis(trimethylsilyl)furan (60) in $80 \%$ yield (Scheme 15). Direct lithiation of 60<smiles>C[As]c1cocc1S(C)(=O)=O</smiles>

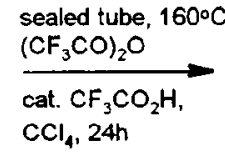

(80\%)

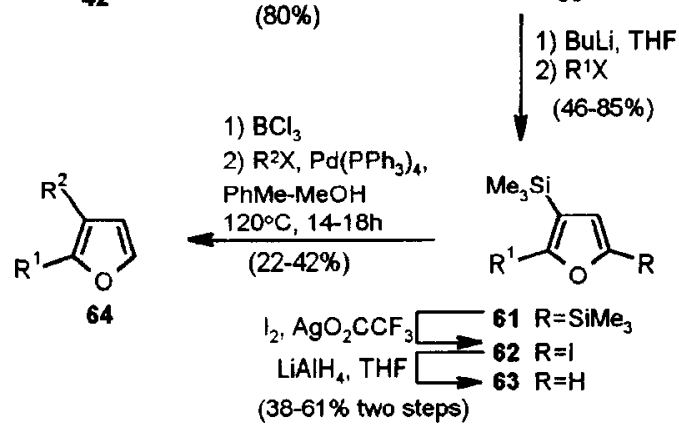

Scheme 15 with BuLi and trapping of the anion with electrophiles, provided furan 61. The TMS group at C-5 could be selectively ipsoiodinated to give 62 . The iodine was easily removed by treatment of $\mathbf{6 2}$ with LAH providing 2,3-disubstituted furan $\mathbf{6 3}$. Finally, 2,3-disubstituted furans $\mathbf{6 4}$ were prepared by using Wong's boroxine protocol followed by a Suzuki coupling.

An alternative strategy was developed towards compounds like 64 as the overall yield of 64 was low starting with 42.28 Furan $\mathbf{6 5}^{23,29}$ was lithiated exclusively at the less hindered C-2 position with $t \mathrm{BuLi}$ (Scheme 16). Trapping of the anion with

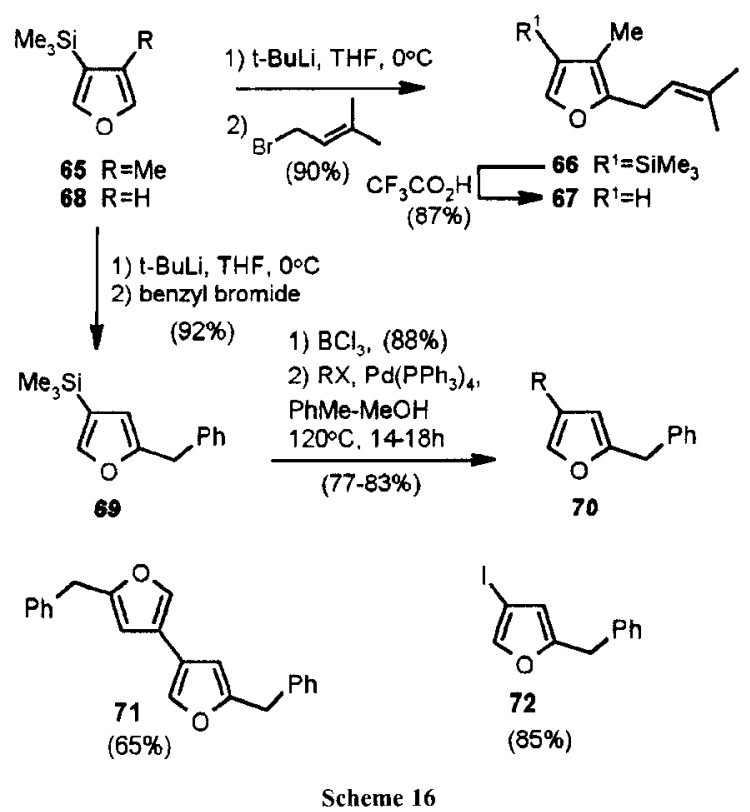

electrophiles yielded 66 , which could be proto-desilylated with trifluoroacetic acid to give $\mathbf{6 7}$ in high overall yield. 

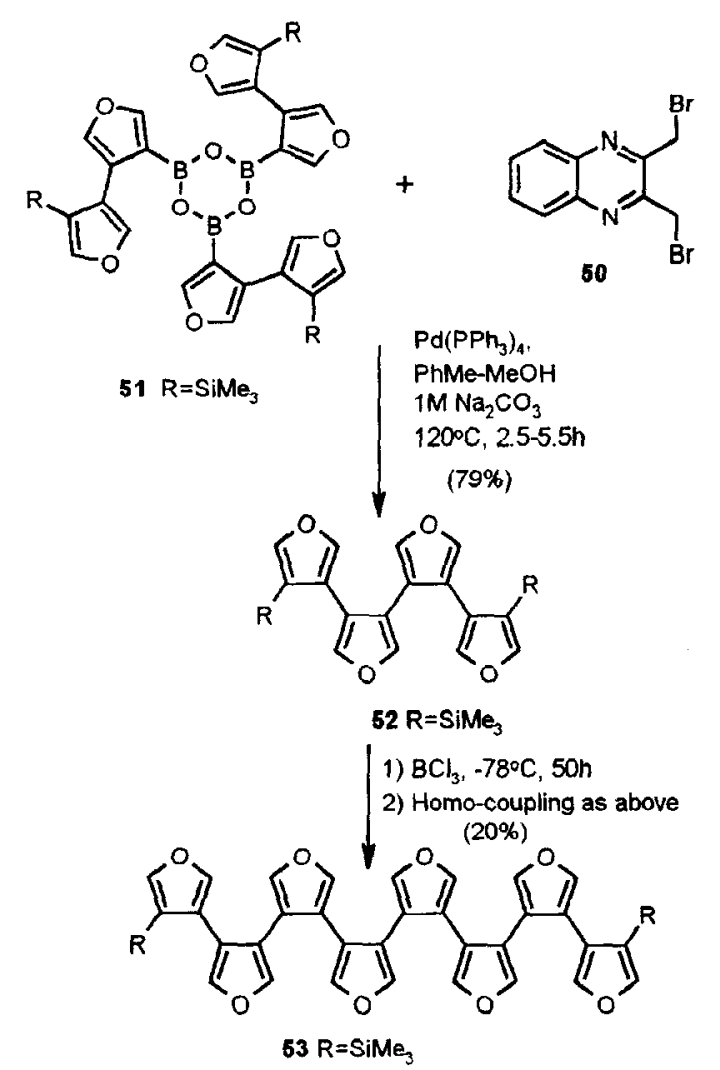

Scheme 13

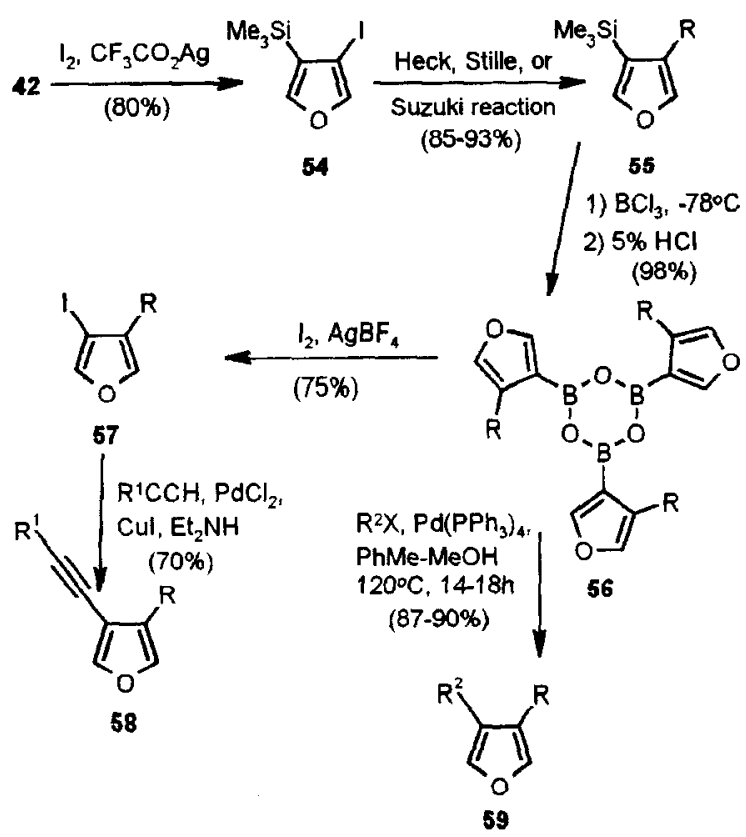

Scheme 14

give 56. Furan 56 could be treated with $I_{2}$ in the presence of $\mathrm{AgBF}_{4}$ to provide iodide 57 , which was used to prepare acetylenic furans like $\mathbf{5 8}$. Or, $\mathbf{5 6}$ could be used directly in a Suzuki coupling to give 3,4-disubstituted furans 59 . The monoreplacement of the trimethylsilyl groups in $\mathbf{4 2}$ clearly expands the scope of the use of furan $\mathbf{4 2}$ in synthesis.
Most recently, Wong and colleagues ${ }^{28}$ have reported new strategies towards the synthesis of 2,3-, 2,4-, 2,3,4-, and $2,3,5$-substituted furan rings. The approach towards the preparation of 2,3-disubstituted furan rings was developed by observing that when furan 42 is heated in a sealed tube at $160^{\circ} \mathrm{C}$ in the presence of trifluoroacetic anhydride containing a catalytic amount of trifluoroacetic acid, one of the silyl groups undergoes a $[1,2] \mathrm{C} \rightarrow \mathrm{C}$ silyl migration to provide 2,4-bis(trimethylsilyl)furan (60) in $80 \%$ yield (Scheme 15). Direct lithiation of 60<smiles>CSc1cocc1[AsH3]</smiles>

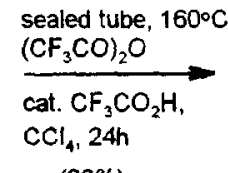

(80\%)<smiles>[R]c1ccoc1C</smiles>

$$
\text { 1) } \mathrm{BCl}_{3}
$$
2) $\mathrm{R}^{2} \mathrm{X}, \mathrm{Pd}\left(\mathrm{PPh}_{3}\right)_{4}$,<smiles>CSc1cc(C)co1</smiles>

60 1) BULi, THF 2) $R^{\prime} X$ $(46-85 \%)$ $\frac{\begin{array}{c}\mathrm{PhMe}-\mathrm{MeOH} \\ 120^{\circ} \mathrm{C}, 14-18 \mathrm{Bh}\end{array}}{(22-42 \%)}$ $\mathrm{Me}_{3} \mathrm{Si}$<smiles>[R]c1cc([As])c([R])o1</smiles>

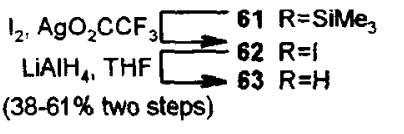

Scheme 15

with BuLi and trapping of the anion with electrophiles, provided furan 61. The TMS group at C-5 could be selectively ipsoiodinated to give $\mathbf{6 2}$. The iodine was easily removed by treatment of $\mathbf{6 2}$ with LAH providing 2,3-disubstituted furan $\mathbf{6 3}$. Finally, 2,3-disubstituted furans 64 were prepared by using Wong's boroxine protocol followed by a Suzuki coupling.

An alternative strategy was developed towards compounds like 64 as the overall yield of 64 was low starting with $42 .{ }^{28}$ Furan $6^{23.29}$ was lithiated exclusively at the less hindered C-2 position with $t$-BuLi (Scheme 16). Trapping of the anion with

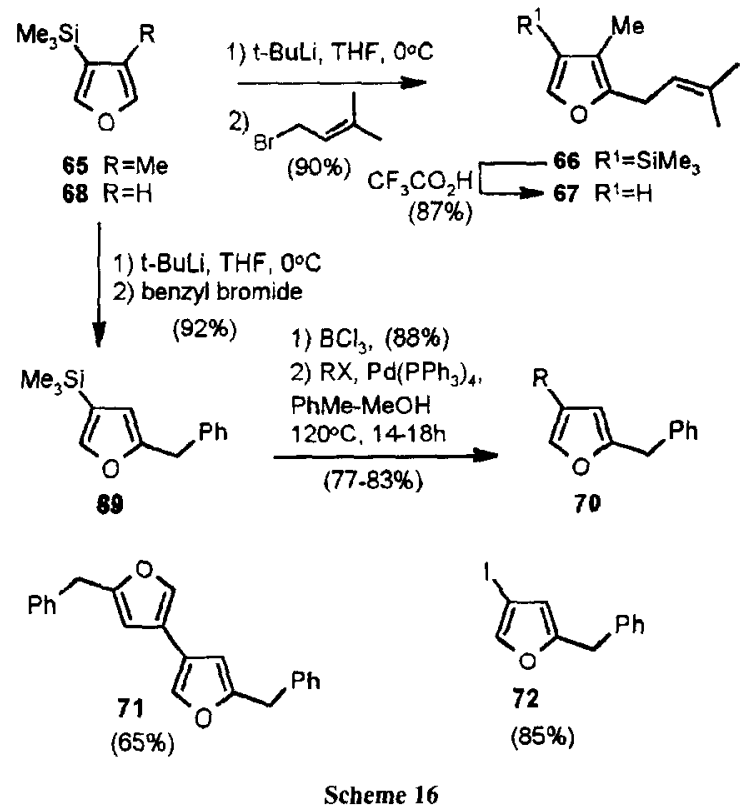

electrophiles yielded 66 , which could be proto-desilylated with trifluoroacetic acid to give $\mathbf{6 7}$ in high overall yield. 
Starting from furan 68, Wong was able to prepare 2,4-disubstituted furans (Scheme 16). 28 Treatment of 68 with $t$-BuLi in THF at $0{ }^{\circ} \mathrm{C}$ and trapping of the anion with benzyl bromide provided only furan 69 in which a regiospecific lithiation at the C-5 position of $\mathbf{6 8}$ had occurred. Using his boroxine chemistry followed by a Suzuki coupling resulted in the preparation of 2,4-disubstituted furans $\mathbf{7 0}$. As might be expected, the boroxine generated and isolated by the treatment of 69 with $\mathrm{BCl}_{3}$ can either be homo-coupled to provide $\mathbf{7 1}$ or iodo-deboronated to provide 72 , which can be used further to prepare other 2,4-disubstituted furan rings.

Compound 54 has been used to prepare 2,3,4-trisubstituted furan rings (Scheme 17).28 A nickel catalyzed cross-coupling

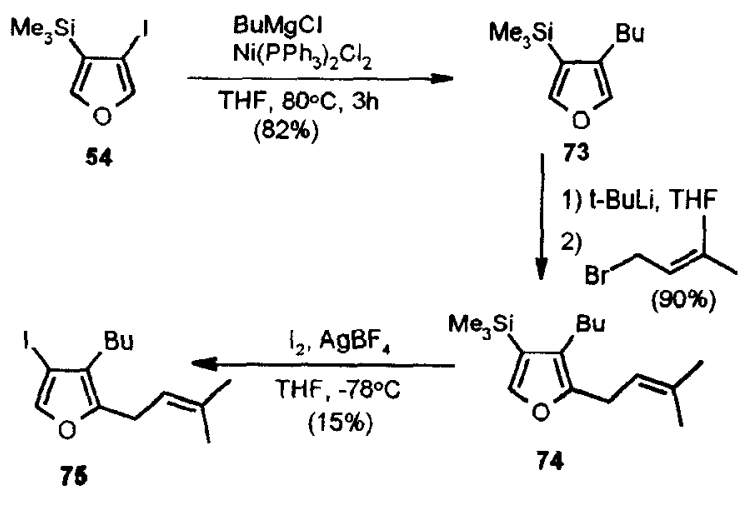

Scheme 17

reaction with butylmagnesium chloride and 54 gave 73 . Regiospecific lithiation of 73 at $\mathrm{C}-2$ with $t$-BuLi and trapping the anion with an electrophile provided $\mathbf{7 4}$ in which the silyl group could be replaced by an iodine atom, albeit in low yield, thereby providing a route to 2,3,4-trisubstituted furans 75 .

Finally, Wong ${ }^{2 x}$ has developed a route to 2,3,5-trisubstituted furans 77 (Scheme 18). Furan 62, prepared according to Scheme

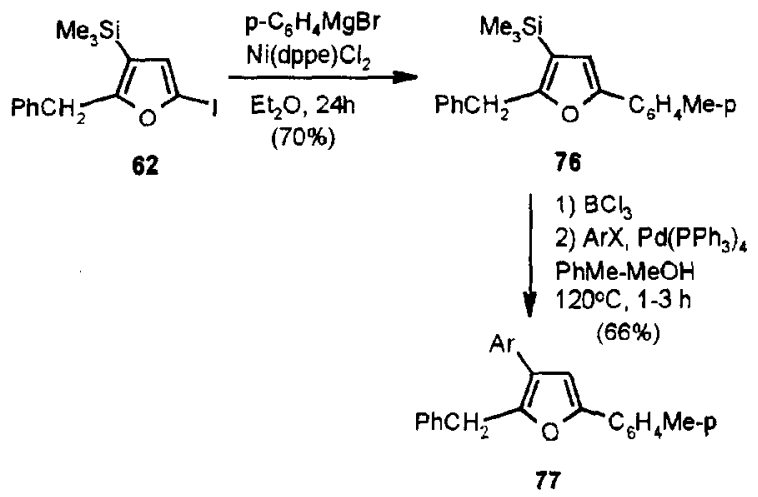

Scheme 18

15 , was treated with $p$-tolylmagnesium bromide in the presence of a nickel catalyst to provide $\mathbf{7 6}$. The trimethylsilyl group in $\mathbf{7 6}$ was then replaced with aromatic rings via Wong's boroxine chemistry, followed by a Suzuki reaction giving 77 . The last few schemes clearly show the important role that a trimethylsilyl group plays in the preparation of a variety of furan substitution patterns. Not only can the silyl group be ipsosubstituted by boron and iodine, but the steric size of the group can help direct lithiation to a site remote (i.e. less hindered) from the silane.

So far the silyl groups have been used for ipso-substitution, directing metalation reactions and removed completely by treatment with TBAF, through a silyl migration, or through a proto-desilylation reaction with acid. An additional use of the trimethylsilyl group is to treat 2-substituted silylated furans with either a peracid or singlet oxygen. This results in a regiospecific conversion of the silylated furan to a butenolide in which the carbonyl group is attached to the carbon atom that the silyl group was initially attached. For example, Kuwajima ${ }^{30}$ reported that furan 78 when treated with peracetic acid formed but3 -enolide 79 in yields ranging from $36-84 \%$ (Scheme 19).

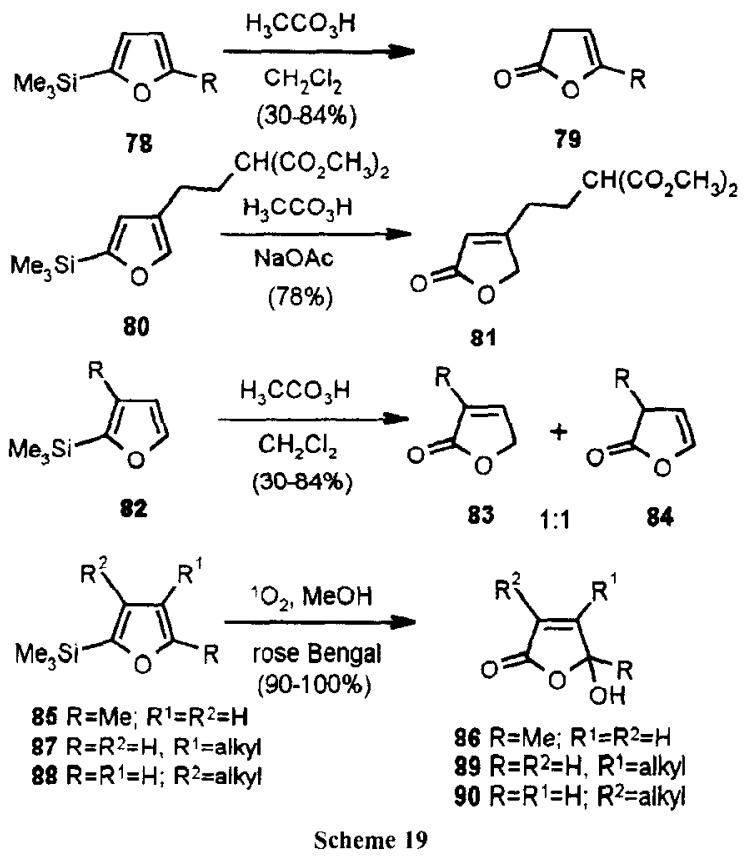

Goldsmith and Liotta ${ }^{31}$ later showed that 2-silylated-4-substituted furan ring 80 also underwent an oxidation reaction with peracetic acid, but provided but-2-enolide $\mathbf{8 1}$ in $78 \%$ yield. Tannis $^{32}$ has also shown that 2-silylated-3-substituted furan rings 82 undergo a similar regiospecific oxidation to give a $1: 1$ mixture of but-2- and -3-enolides 83 and 84 in $78 \%$ yield. Adam and Rodriguez ${ }^{33}$ have reported that 5-substituted-2-(trimethylsilyl)furan 85 can be oxidized to 4-hydroxy-4-substitutedbut-2-enolide 86 in quantitative yield. Similarly, 3- or 4-substituted-2-(trimethylsilyl)furans 87 and 88 can be oxidized regiospecifically to 2 - or 3-substituted-4-hydroxy-but-2-enolides 89 and 90 respectively in yields ranging from $89-94 \% .34$

\section{Conclusions}

This brief review clearly shows the important role that silicon has played in the development of new strategies for the preparation of multi-substituted furan rings. The size, migratory aptitude, tendency to undergo ipso-substitutions and their ease of attachment to and removal from furan systems of silyl groups has been used to the fullest. Some future endeavors might include using fluorinated silanes so that they can be directly replaced by other functionalities using palladium-coupling reactions. ${ }^{35}$ This would eliminate the need for an ipsoreplacement of the silane with an iodine or boron atom. Very little work has been done with the more robust tert-butyldimethylsilyl group. Methods for its replacement with other functionalities, rather than just removing it, would offer additional alternatives to those involving the trimethylsilyl group. 


\section{References}

I B. A. Kcay and P. W. Dibblc, in Comprehensive Heterocyclic Chemistry II, ed., C. W. Bird, Elsevier, New York, 1996, vol. 2, ch. 2.08, pp $395-436$.

2 X. L. Hou, H. Y. Chcung, T. Y. Hon, P. L. Kwan, T. H. Lo, S. Y. Tong and H. N. C. Wong, Tetrahedron, 1998, 54, 1955 and references therein.

3 R. A. Benkeser and R. B. Currie, J. Am. Chem. Soc., 1948, 70, 1780.

4 A. J. Carpenter and D. J. Chadwick, Tetrahedron Lett., 1985, 26 1777

5 D. W. Knight and A. P. Nott, J. Chem. Soc., Perkin Trans. 1, 1983, 791.

6 D. J. Chadwick, M. V. McKnight and R. Ngochindo, J. Chem. Soc. Perkin Trans. 1, 1982, 1343.

7 D. S. Ennis and T. L. Gilchrist, Tetrahedron Lett., 1990, 46, 2623.

8 J.-Y. Lenoir, P. Ribereau and G. Queguiner, J. Chem. Soc., Perkin Trans. I, 1994, 2943.

9 E. Bures, P. G. Spinazze, G. Beese, I. R. Hunt, C. Rogers and B. A. Keay, J. Org. Chem., 1997, 62, 8741.

10 E. Bures, J. A. Nicman, S. Y'u, P. G. Spinazze, J.-L. J. Bontront, I. R Hunt, A. Rauk and B. A. Kcay, J. Org. Chem., 1997, 62, 8750.

11 R. E. Danso-Danquah, A. I. Scott and D. Becker, Tetrahedron, 1993, 49, 8195.

12 J. A. Nicman and B. A. Keay, Tetrahedron Lett., 1994, 35, 5335.

13 E. J. Bures and B. A. Kcay, Tetrahedron Lett. 1988, $29,1247$.

14 S. Katsumura, K. lchikawa and H. Mori, Chem. Lett, 1993, 1525.

15 R. L. Beddocs, M. L. Lewis, P. Gilbert. P. Quayle, S.P. Thompson, S. Wang and K. Mills, Tetrahedron Lett., 1996, 37, 9119.

16 B. A. Keay and J.-L. Bontront, Can. J. Chem., 1991, 69, 1326.
17 W. A. Cristofoli and B. A. Keay, Tetrahedron Lett., 1991, 32,5881

18 S. P. Maddaford and B. A. Keay, J. Org. Chem., 1994, 59, 6501.

19 S. P. Maddaford, N. G. Andersen, W.A. Cristofoli and B.A. Keay, J. Am. Chem. Soc., 1996, 118, 10766.

$20 \mathrm{~K}$. Nakayama, Y. Harigaya, H. Okamoto and A. Tanaka, $J$. Heterocycl. Chem, 1991, 28, 853

21 K. Nakayama and A. Tanaka, Chem. Pharm. Bull., 1992, 40, 1966.

22 K. Nakayama and A. Tanaka, Chem. Express, 1991, 6, 699.

23 M. S. Ho and H. N. C. Wong J. Chem. Soc., Chem. Commun., 1989, 1238.

24 Z. Z. Song, Z. Y. Zhou, T. C. W. Mak and H. N. C. Wong, Angew Chem., Int. Ed, Engl., 1993, 32, 432

25 Z. Z. Song, M. S. Ho and H. N. C. Wong, J. Org. Chem., 1994, 59 3917.

26 Z. Zhong and H. N. C. Wong, J. Org. Chem., 1994, 59, 33.

27 Z. Z. Song and H. N. C. Wong, Liebigs Ann. Chem., 1994, 29.

28 M. K. Wong, C. Y. Leung and H. N. C. Wong, Tetrahedron, 1997, 53, 3497.

29 Z. Z. Song and H. N. C. Wong, J. Chin. Chem. Soc., 1995, 42, 673.

30 I. Kuwajima and H. Urabe, Tetrahedron Lett., 1981, 22, 5191.

31 D. Goldsmith, D. Liotta, M. Saindane, L. Waykole and P. Brown, Tetrahedron Lett. 1983, 24, 5835 .

32 S. P. Tannis and D. Head, Tetrahedron Lett., 1984, 25, 4451

33 W. Adam and A. Rodriguez, Tetrahedron Lett., 1981, 22, 3505.

34 S. Katsumura, K. Hori, S. Fujiwara and S. Isoc, Tetrahedron Lett., 1985, 26, 4625 and Tetrahedron Lett., 1988, 29, 1173.

35 Y. Hatanaka, S. Fukushima and T. Hiyama, Tetrahedron, 1992, 48, 2113 and references thercin.

Review $8 / 09439 J$ 